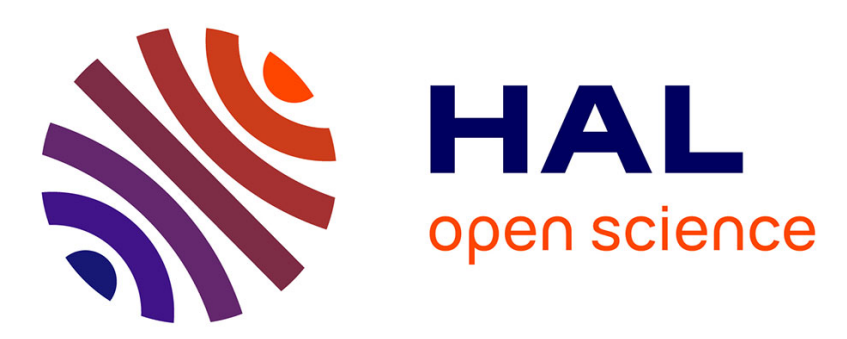

\title{
Long-time anomalous swimmer diffusion in smectic liquid crystals
}

Claudia Ferreiro-Córdova, John Toner, Hartmut Löwen, Henricus H Wensink

\section{To cite this version:}

Claudia Ferreiro-Córdova, John Toner, Hartmut Löwen, Henricus H Wensink. Long-time anomalous swimmer diffusion in smectic liquid crystals. Physical Review E , 2018, 97, pp.062606. 10.1103/PhysRevE.97.062606 . hal-02322628

\section{HAL Id: hal-02322628 \\ https://hal.science/hal-02322628}

Submitted on 21 Oct 2019

HAL is a multi-disciplinary open access archive for the deposit and dissemination of scientific research documents, whether they are published or not. The documents may come from teaching and research institutions in France or abroad, or from public or private research centers.
L'archive ouverte pluridisciplinaire HAL, est destinée au dépôt et à la diffusion de documents scientifiques de niveau recherche, publiés ou non, émanant des établissements d'enseignement et de recherche français ou étrangers, des laboratoires publics ou privés. 


\title{
Long-time anomalous swimmer diffusion in smectic liquid crystals
}

\author{
Claudia Ferreiro-Córdova, ${ }^{1}$ John Toner, ${ }^{2}$ Hartmut Löwen, ${ }^{3}$ and Henricus H. Wensink ${ }^{1, *}$ \\ ${ }^{1}$ Laboratoire de Physique des Solides, CNRS, Université Paris-Sud, Université Paris-Saclay, 91405 Orsay, France \\ ${ }^{2}$ Department of Physics and Institute of Theoretical Science, University of Oregon, Eugene, Oregon 97403, USA \\ ${ }^{3}$ Institut für Theoretische Physik II: Weiche Materie, Heinrich-Heine-Universität Düsseldorf, \\ Universitätsstraße 1, 40225 Düsseldorf, Germany
}

(Received 10 May 2018; published 29 June 2018)

\begin{abstract}
The dynamics of self-locomotion of active particles in aligned or liquid crystalline fluids strongly deviates from that in simple isotropic media. We explore the long-time dynamics of a swimmer moving in a three-dimensional smectic liquid crystal and find that the mean-square displacement transverse to the director exhibits a distinct logarithmic tail at long times. The scaling is distinctly different from that in an isotropic or nematic fluid and hints at the subtle but important role of the director fluctuation spectrum in governing the long-time motility of active particles. Our findings are based on a generic hydrodynamic theory and Brownian dynamics computer simulation of a three-dimensional soft mesogen model.
\end{abstract}

DOI: 10.1103/PhysRevE.97.062606

\section{INTRODUCTION}

The main focus of research in the active matter physics community has recently shifted towards studying microswimming through complex media that can no longer be represented by a simple isotropic Newtonian continuum [1-5]. The main motivation stems from the fact that many microorganisms operate in crowded environments with a nonuniform positional and/or orientational microstructure that may generate liquid crystalline or viscoelastic properties. Examples encompass the dynamics of cilia and spermatozoa in mucus [6,7], of bacteria migrating through tissue [8], or through complex extracellular matrices such as in biofilms and the motion of nematodes residing in soil [9].

These real-life situations call for more sophisticated models for microswimming that aim at a better understanding of the complexity of the medium, such as in the case of viscoelastic fluids [10-20] or liquid crystalline fluids [18,21-30]. A similar increase in medium complexity is attained by considering active locomotion around random or patterned obstacles [31-33] and by studying the role of active dopants in crystalline host systems [34-36].

With most approaches thus far focusing on collective properties or on short-term swimmer motility, we wish to address the impact of liquid crystalline order on the long-time diffusive behavior of such a swimmer. In a previous paper [23], we have undertaken such a study by focusing on active diffusion through a simple Lebwohl-Lasher lattice nematic. Here we wish to build upon these findings and consider a more appropriate off-lattice model to explore long-time active diffusion in lamellar or smectic systems which possess a distinct unidimensional long-range periodicity imparted by "stacked" membranes, each with a quasibidimensional liquid-like internal order. In contrast to the simplified lattice

\footnotetext{
*rik.wensink@u-psud.fr
}

representation of a liquid crystal, the off-lattice model enables us to vary the positional symmetry of the host medium simply by changing the system temperature. The model thus offers a route to sampling the nontrivial swimmer dynamics moving through a range of different host phases and use temperature as a control parameter. We find that in the case of nematic and smectic hosts, the swimmer MSD perpendicular to the director is nontrivial and obeys a distinct logarithmic scaling with time. Specifically, we find that the mean-squared lateral wandering $\left\langle\left(\left[\Delta \mathbf{r}_{s}^{\perp}(t)\right]^{2}\right\rangle\right.$ of a swimmer in a smectic obeys

$$
\left\langle\left[\Delta \mathbf{r}_{s}^{\perp}(t)\right]^{2}\right\rangle= \begin{cases}\bar{D}_{s} t \ln \left(\frac{t}{t_{0}}\right), & t \ll t_{a} \\ \bar{D}_{s} t \sqrt{\ln \left(\frac{t}{t_{0}}\right)}, & t \gg t_{a},\end{cases}
$$

where $\bar{D}_{s}$ represents an anomalous diffusion constant and $t_{a}$ is a system-dependent crossover time that is extremely sensitive to both the speed of the swimmer and the parameters of the smectic liquid crystal. An explicit expression for $t_{a}$ is given in Eqs. (57) and (58).

In contrast, it was shown in Ref. [23] that a swimmer in a nematic also exhibits anomalous diffusion but obeys the law

$$
\left\langle\left[\Delta \mathbf{r}_{s}^{\perp}(t)\right]^{2}\right\rangle=\bar{D}_{n} t \ln \left(\frac{t}{t_{0}}\right) .
$$

Since the typical scaling exponent for a smectic fluid differs from that of a nematic system, measurement of the swimmer MSD transverse to the main director of the host could be used to probe structural features of the medium, in particular the presence of local lamellar order. Of course, to observe this difference, experiments must probe times $t \gg t_{a}$, since the scaling for shorter times is the same in both the smectic and nematic phases. In many experimental systems, this will be quite difficult: because of the exponential dependence of the crossover time $t_{a}$ (57) on material parameters and swimming speed, this time will be literally astronomical in many cases [see the estimates after Eq. (58) below]. The best hope of seeing 
the $t \gg t_{a}$ limit of (1) is for very fast swimmers $\left(v \sim 50 \frac{\mu}{\mathrm{sec}}\right)$ in very high dilution lyotropic smectics [see the estimate (60) below for such smectics].

In our simulations, we circumvent this difficulty by simulating non-momentum-conserving dynamics, which, although unphysical for real experiments, reduces the crossover time to extremely small values. The asymptotic form of (1) for $t \gg t_{a}$ is unchanged by this change in the dynamics; it is simply reached at much shorter times. And, indeed, these simulations agree with our theoretical predictions, which underscores the fundamental impact of director fluctuations in steering swimmers through liquid crystalline backgrounds.

The rest of this paper is structured as follows. In Sec. II we briefly recapitulate our hydrodynamic theory of swimmer motility in anisotropic media and provide results for the longtime swimmer dynamics in a three-dimensional smectic phase. These predictions are generic and are valid for swimmers in both thermotropic and lyotropic smectic hosts. The predictions are tested against Brownian dynamics computer simulations based on a soft-nematogen model which is described in detail in Sec. III. In the last section, we formulate the main conclusions of our study.

\section{MODEL FOR LONG-TIME SWIMMER MOTILITY}

In very close analogy with earlier treatments of swimmers in nematics [23], we will consider a self-propelled swimmer moving through an otherwise equilibrium, ordered smectic A. This swimmer has no memory, or, at best, only a short-term memory, of its past direction of motion. Furthermore, the dynamics of the entire system (smectic plus swimmer) are rotation invariant: that is, the swimmer carries no internal "compass"; any preference it exhibits for one direction of motion over any other must arise from the local layer normal $\hat{\mathbf{n}}\left[\mathbf{r}_{s}(t)\right]$ at the current location $\mathbf{r}_{s}(t)$ of the swimmer. This requirement of locality arises from the physically reasonable assumption that the interactions of the swimmer with the surrounding smectic are short-ranged in space.

The average value of the instantaneous velocity $d \mathbf{r}_{s}(t) / d t$ of such a swimmer must be along $\hat{\mathbf{n}}\left[\mathbf{r}_{s}(t)\right]$; rotation invariance plus locality allow no other direction $\left[\operatorname{except}-\hat{\mathbf{n}}\left[\mathbf{r}_{s}(t)\right]\right.$; we will discuss this option below]. Hence, the instantaneous velocity $d \mathbf{r}_{s}(t) / d t$ must be given by

$$
\frac{d \mathbf{r}_{s}(t)}{d t}=v_{s} \hat{\mathbf{n}}\left(\mathbf{r}_{s}(t), t\right)+\mathbf{f}(t),
$$

where $\mathbf{f}(t)$ is a zero mean random fluctuation in the velocity, and $v_{s}$ is the mean speed of the swimmer. Note that in general $v_{s} \neq v_{s}^{0}$, where $v_{s}^{0}$ is the "bare," or instantaneous, speed of the swimmer, due to the effects of fluctuations. Indeed, in general, we expect $v_{s}<v_{s}^{0}$. In practice, $v_{s}$ can be determined only by measuring the mean motion of the swimmer over long times; this will be discussed in more detail below.

While we have motivated (3) purely on symmetry grounds, there is certainly at least one physical mechanism to generate such alignment of a swimmer with the director: anchoring of the director on the surface of the swimmer, which would lead to torques tending to align the swimmer with the director.

The statistics of the fluctuations $\mathbf{f}$ are also almost completely determined by the requirements of rotation invariance and locality in space and time. In a "coarse-grained" theory, in which we imagine having averaged our dynamics over timescales long compared to the time of individual molecular "kicks" experienced by the swimmer, but short compared to the timescales we wish to investigate, $\mathbf{f}$ can be thought of as a sum of a large number of random molecular kicks at different microscopic times, which are therefore statistically independent. The central limit theorem then tells us that the statistics of $\mathbf{f}$ should be Gaussian. Its statistics are then completely specified by its two-point correlations with the local layer normal $\hat{\mathbf{n}}(\mathbf{r}, t)$ and itself; rotation invariance and spatio-temporal locality imply that these are given by

$$
\begin{aligned}
\left\langle f_{\alpha}(t) f_{\beta}\left(t^{\prime}\right)\right\rangle= & 2 \Delta_{I} \delta_{\alpha \beta} \delta\left(t-t^{\prime}\right)+2 \Delta_{A} n_{\alpha}\left(\mathbf{r}_{s}(t), t\right) \\
& \times n_{\beta}\left(\mathbf{r}_{s}(t), t\right) \delta\left(t-t^{\prime}\right)
\end{aligned}
$$

and

$$
\left\langle f_{\alpha}(t) n_{\beta}\left(\mathbf{r}_{s}\left(t^{\prime}\right), t^{\prime}\right)\right\rangle=2 \Delta_{f n} \delta_{\alpha \beta} \delta\left(t-t^{\prime}\right),
$$

where $\alpha$ and $\beta$ are Cartesian indices, and $\Delta_{I}, \Delta_{A}$, and $\Delta_{f n}$ are phenomenological parameters which set the size of the fluctuations of the swimmer. Because the swimmer is a nonequilibrium agent, these parameters do not, in general, satisfy any kind of Einstein relation; that is, they are independent parameters.

The model just described neglects "hairpin turns": fluctuations in which the swimmer reverses its direction of motion relative to the local layer normal (that is, where it makes an angle of more than $90^{\circ}$ with the director. If such turns occur, then at times large compared to the timescale for hairpin turns, the MSD should turn purely diffusive (albeit with an anisotropic diffusivity). However, as discussed in Ref. [23], such turns are strongly suppressed if the "energy barrier" $\Delta E$ against a reversal of the swimmer direction of motion (that is, the energy cost of the swimmer making an angle of $90^{\circ}$ with the local layer normal) is large compared to the thermal energy; i.e., if $\Delta E \gg k_{\mathrm{B}} T$. We have chosen our parameters to ensure this condition in our simulations. Indeed, we have never observed a hairpin turn in our simulations. More importantly, we also expect that in many real experiments, deep within the smectic phase and for a strongly aligned swimmer, $\Delta E \gg k_{\mathrm{B}} T$, so hairpins should be rare, if not nonexistent, as well.

We now proceed to analyze the implications of this theory for the motion of the swimmer. We will start with the mean motion. Taking the average of Eq. (3), and recalling that $\langle\mathbf{f}\rangle=$ $\mathbf{0}$, we immediately obtain an expression for the mean position of the swimmer:

$$
\left\langle\mathbf{r}_{s}(t)\right\rangle=v_{s} t\langle\hat{\mathbf{n}}\rangle \equiv v_{z} t \hat{\mathbf{z}},
$$

where we have taken the mean direction of the layer normal $\hat{\mathbf{n}}$ to be along $\hat{\mathbf{z}}$, and the mean swimmer speed in the $z$ direction is given by $v_{z}=v_{s}|\langle\hat{\mathbf{n}}\rangle|$. Thus, the mean motion of the swimmer is purely ballistic. Note that the speed $v_{z}$ of this motion is not $v_{s}$, due to the fact that fluctuations reduce $\langle\hat{\mathbf{n}}\rangle$ below 1 . Indeed, the speed $v_{z}$ can not even be predicted by the continuum theory developed below, since the fluctuations which dominate this reduction are predominantly short wavelength and therefore not accurately described by the continuum, long-wavelength hydrodynamic theory of smectics. Nonetheless, we have still 
made a universal scaling prediction: the mean motion of the swimmer is ballistic, as shown by Eq. (6).

We now turn to the fluctuations about this mean. Consider first the mean-squared lateral displacement of the swimmer:

$$
\left\langle\left[\Delta \mathbf{r}_{s}^{\perp}(t)\right]^{2}\right\rangle \equiv\left\langle\left|\mathbf{r}_{s}^{\perp}(t)-\mathbf{r}_{s}^{\perp}(0)\right|^{2}\right\rangle,
$$

perpendicular to the mean director of the smectic. Here and throughout this paper, $\perp$ and $z$ denote directions perpendicular to and along the layer normal, respectively.

Using the projection of our equation of motion (3) perpendicular to the mean layer normal direction $\hat{\mathbf{z}}$, which reads

$$
\frac{d \mathbf{r}_{s}^{\perp}(t)}{d t}=v_{s} \mathbf{n}_{\perp}\left(\mathbf{r}_{s}, t\right)+\mathbf{f}_{\perp},
$$

and integrating over time gives

$$
\Delta \mathbf{r}_{s}^{\perp}(t) \equiv \mathbf{r}_{s}^{\perp}(t)-\mathbf{r}_{s}^{\perp}(0)=\int_{0}^{t} d t^{\prime}\left[v_{s} \mathbf{n}_{\perp}\left(\mathbf{r}_{s}, t^{\prime}\right)+\mathbf{f}_{\perp}\left(t^{\prime}\right)\right] .
$$

Squaring this, and averaging, we find that $\left\langle\left[\Delta \mathbf{r}_{s}^{\perp}(t)\right]^{2}\right\rangle$ is given by

$$
\begin{aligned}
\left\langle\left[\Delta \mathbf{r}_{s}^{\perp}(t)\right]^{2}\right\rangle= & \int_{0}^{t} d t^{\prime} \int_{0}^{t} d t^{\prime \prime}\left[v_{s}^{2}\left\langle\mathbf{n}_{\perp}\left(\mathbf{r}_{s}\left(t^{\prime}\right), t^{\prime}\right) \cdot \mathbf{n}_{\perp}\left(\mathbf{r}_{s}\left(t^{\prime \prime}\right), t^{\prime \prime}\right)\right\rangle\right. \\
& \left.+2 v_{s}\left\langle\mathbf{n}_{\perp}\left(\mathbf{r}_{s}\left(t^{\prime}\right), t^{\prime}\right) \cdot \mathbf{f}_{\perp}\left(t^{\prime \prime}\right)\right\rangle+\left\langle\mathbf{f}_{\perp}\left(t^{\prime}\right) \cdot \mathbf{f}_{\perp}\left(t^{\prime \prime}\right)\right\rangle\right] .
\end{aligned}
$$

Using the expressions (4) and (5) for the two-point correlations of the Gaussian random velocity, we can immediately evaluate the last two terms, denoted by $I_{2}$ and $I_{3}$, respectively. The first of them is

$$
I_{2}=\int_{0}^{t} d t^{\prime} \int_{0}^{t} d t^{\prime \prime} 2 v_{s}\left\langle\mathbf{n}_{\perp}\left(\mathbf{r}_{s}\left(t^{\prime}\right), t^{\prime}\right) \cdot \mathbf{f}_{\perp}\left(t^{\prime \prime}\right)\right\rangle=6 \Delta_{f n} t
$$

while the second is

$$
I_{3}=\int_{0}^{t} d t^{\prime} \int_{0}^{t} d t^{\prime \prime}\left\langle\mathbf{f}_{\perp}\left(t^{\prime}\right) \cdot \mathbf{f}_{\perp}\left(t^{\prime \prime}\right)\right\rangle=\left[6 \Delta_{I}+2 \Delta_{A}\right] t .
$$

Both of these terms are extremely boring: their contribution to the mean-squared lateral wandering $\left\langle\left[\Delta \mathbf{r}_{s}^{\perp}(t)\right]^{2}\right\rangle$ is simply conventionally diffusive: that is, proportional to time $t$. The anomalous diffusion that we predict comes entirely from the first term in Eq. (10):

$$
I_{1}=v_{s}^{2} \int_{0}^{t} d t^{\prime} \int_{0}^{t} d t^{\prime \prime}\left\langle\mathbf{n}_{\perp}\left(\mathbf{r}_{s}\left(t^{\prime}\right), t^{\prime}\right) \cdot \mathbf{n}_{\perp}\left(\mathbf{r}_{s}\left(t^{\prime \prime}\right), t^{\prime \prime}\right)\right\rangle .
$$

Because the smectic dynamics are invariant under space and time translations, the general director two-point correlation function depends only on the differences of the space and time coordinates:

$$
C_{\perp} \equiv\left\langle\mathbf{n}_{\perp}\left(\mathbf{r}^{\prime}, t^{\prime}\right) \cdot \mathbf{n}_{\perp}\left(\mathbf{r}^{\prime \prime}, t^{\prime \prime}\right)\right\rangle=C_{\perp}\left(\mathbf{r}^{\prime}-\mathbf{r}^{\prime \prime}, t^{\prime}-t^{\prime \prime}\right) .
$$

Now, in Eq. (13), we need this correlation function evaluated when $\mathbf{r}^{\prime}=\mathbf{r}_{s}\left(t^{\prime}\right)$ and $\mathbf{r}^{\prime \prime}=\mathbf{r}_{s}\left(t^{\prime \prime}\right)$. These vectors are given by

$$
\mathbf{r}_{s}(t)=\mathbf{r}_{s}(0)+v_{z} t \hat{\mathbf{z}}+\Delta \mathbf{r}_{s}^{\perp}(t) .
$$

To proceed further, we need to calculate this director correlation, which is independent of the dynamics of the swimmer but clearly does depend on the dynamics of the smectic. However, we will show, in the final subsection of this theoretical section, that in fact the motion of the swimmer is, at sufficiently long times, independent of the smectic dynamics, provided only that those dynamics do relax back to thermal equilibrium.

However, that phrase "sufficiently long times" is highly loaded: for many real experimental systems, the time that must be reached before the asymptotic law for the lateral superdiffusion of the swimmer that we find below holds is astronomical. Fortunately, for shorter timescales, the behavior is still superdiffusive but with a different scaling law. All of this will be discussed in the final subsection of this theoretical section.

In the next subsection, we will obtain the asymptotic superdiffusive scaling law that applies for all dynamical models.

\section{A. Universal asymptotic superdiffusion}

We will show in the next subsection that at very long times, the correlation function $C_{\perp}\left(\mathbf{r}^{\prime}-\mathbf{r}^{\prime \prime}, t^{\prime}-t^{\prime \prime}\right)$, when evaluated at typical values of $\mathbf{r}^{\prime}=\mathbf{r}_{s}\left(t^{\prime}\right)$ and $\mathbf{r}^{\prime \prime}=\mathbf{r}_{s}\left(t^{\prime \prime}\right)$, is well approximated by its equal time value:

$$
C_{\perp}\left(\mathbf{r}_{s}\left(t^{\prime}\right)-\mathbf{r}_{s}\left(t^{\prime \prime}\right), t^{\prime}-t^{\prime \prime}\right) \approx C_{\perp}\left(\mathbf{r}_{s}\left(t^{\prime}\right)-\mathbf{r}_{s}\left(t^{\prime \prime}\right), 0\right) .
$$

However, we will also show in the next subsection that for many realistic experimental systems, this "equal-time" approximation holds only for astronomically long timescales. For shorter timescales, we still predict superdiffusive behavior, but with a different scaling law. Our simulations, however, are done for a model and in a regime in which this asymptotic behavior is reached at quite short times. For this section, we will simply assume that this equal time approximation (16) holds and investigate its consequences for the lateral diffusion of the swimmer.

The simplification provided by the equal time approximation is that equal time correlations can be calculated from equilibrium Boltzmann statistics. Indeed, many different dynamical models will relax back to the same equilibrium Boltzmann distribution, and, therefore, the same equal time correlation functions. This is particularly relevant for the simulations we perform here, since we simulated a model without momentum conservation, whereas any real bulk three-dimensional smectic will, of course, have momentum conservation [37]. We will not consider the motion of a swimmer in such systems here. Fortunately, since our model (by construction) relaxes back to the equilibrium state of a smectic $\mathrm{A}$, it is guaranteed to have the same equal time correlation functions as the more realistic momentum-conserving models that describe the experimentally relevant momentum-conserving case.

We will limit ourselves to the case in which the boundary conditions allow the smectic to assume its energetically optimal configuration: evenly spaced parallel layers. For example, homeotropic boundary conditions between two parallel palates could accomplish this. Boundary conditions that induced smectic textures like focal conics, or topological defects like dislocations in grain boundaries, should be avoided. Having reduced our problem to the calculation of equilibrium, equaltime correlations of the layer normal in a smectic A, we now proceed to calculate those correlations. This quite standard calculation starts with the observation that, in a smectic A, the layer normal $\hat{\mathbf{n}}(\mathbf{r}, t)$ is determined entirely by the smectic layer displacement field $u(\mathbf{r}, t)$ through the simple geometrical 
relation [38]

$$
\hat{\mathbf{n}}(\mathbf{r}, t) \approx \hat{\mathbf{z}}-\nabla_{\perp} u(\mathbf{r}, t),
$$

where the approximate equality holds to linear order in $\nabla_{\perp} u(\mathbf{r}, t)$. Fourier transforming this relation in space then implies

$$
\left\langle\left|\mathbf{n}_{\perp}(\mathbf{q}, t)\right|^{2}\right\rangle=q_{\perp}^{2}\left\langle|u(\mathbf{q}, t)|^{2}\right\rangle .
$$

The calculation of the $u-u$ correlation function in this expression from equilibrium Boltzmann statistics requires only a knowledge of the equilibrium elastic Hamiltonian for layerpositional fluctuations $u(\mathbf{r})$. This is well known to be [38,39]

$$
H=\frac{1}{2} \int d^{3} r\left[B\left(\partial_{z} u\right)^{2}+K\left(\nabla_{\perp}^{2} u\right)^{2}\right] .
$$

From this model, it is straightforward to derive the required $u-u$ correlation function in Ref. (18) by Fourier transforming and applying equipartition. This gives the standard result [38]

$$
\left\langle|u(\mathbf{q}, t)|^{2}\right\rangle=\frac{k_{B} T}{G_{\mathbf{q}}},
$$

where we have defined

$$
G_{\mathbf{q}} \equiv K q_{\perp}^{4}+B q_{z}^{2}
$$

Using this in (18) gives the director correlations in Fourier space

$$
\left\langle\left|\mathbf{n}_{\perp}(\mathbf{q}, t)\right|^{2}\right\rangle=\frac{k_{B} T q_{\perp}^{2}}{G_{\mathbf{q}}},
$$

which in turn implies that real space director fluctuations are given by

$$
\begin{aligned}
C_{\perp}(\mathbf{r}, 0) & =\left\langle\mathbf{n}_{\perp}(\mathbf{r}+\mathbf{R}, t) \cdot \mathbf{n}_{\perp}(\mathbf{R}, t)\right\rangle \\
& =k_{B} T \int \frac{d^{3} q}{8 \pi^{3}} \frac{q_{\perp}^{2} e^{i \mathbf{q} \cdot \mathbf{r}}}{G_{\mathbf{q}}} .
\end{aligned}
$$

Using this expression in our equal time approximation (16), and using that in turn in expression (14) for the nematic correlations and (13) for the superdiffusive integral $I_{1}$, we obtain

$$
I_{1}=v_{s}^{2} \int_{0}^{t} d t^{\prime} \int_{0}^{t} d t^{\prime \prime} C\left(t^{\prime}-t^{\prime \prime}\right),
$$

where we have defined

$$
C(\delta t) \equiv C_{\perp}\left(\mathbf{r}_{s}(t+\delta t)-\mathbf{r}_{s}(t), 0\right),
$$

with $r_{s}(t)$ given by (15). Using (23) in this expression and performing the integral over $q_{z}$ by complex contour techniques gives

$$
\begin{aligned}
C(\delta t) & =\left\langle\mathbf{n}_{\perp}\left(\Delta \mathbf{r}_{s}^{\perp}(\delta t)+v_{s} \delta t \hat{\mathbf{z}}, 0\right) \cdot \mathbf{n}_{\perp}(\mathbf{0}, 0)\right\rangle \\
& =k_{B} T \int \frac{d^{2} q_{\perp}}{8 \pi^{2} \sqrt{B K}} e^{i \mathbf{q}_{\perp} \cdot \Delta \mathbf{r}_{s}^{\perp}(\delta t)-v_{s} \lambda q_{\perp}^{2}|\delta t|},
\end{aligned}
$$

where $\lambda \equiv \sqrt{K / B}$ is the familiar smectic penetration length [38], and we have defined $\Delta \mathbf{r}_{s}^{\perp}(\delta t) \equiv \mathbf{r}_{s}(t+\delta t)-\mathbf{r}_{s}(t)$. Doing the simple Gaussian integrals over the two components of $\mathbf{q}_{\perp}$ gives

$$
C(\delta t)=\frac{k_{B} T}{8 \pi v_{s} K|\delta t|}\left\langle\exp \left[-\frac{\left[\Delta \mathbf{r}_{s}^{\perp}(\delta t)\right]^{2}}{4 \lambda v_{s}|\delta t|}\right]\right\rangle .
$$

Noting that each of the two components $\Delta x, \Delta y$ of $\Delta \mathbf{r}_{s}^{\perp}(\delta t)$ is a zero-mean Gaussian random variable (since it is the sum of Gaussian random variables, as it is linearly related to the noise, which is Gaussian, and the director $\hat{\mathbf{n}}$, whose fluctuations are also Gaussian), and using the result for a zero mean Gaussian random variable $x$ that $\left\langle\exp \left(-k x^{2}\right)\right\rangle=1 / \sqrt{1+2 k\left\langle x^{2}\right\rangle}$, we get

$$
\begin{aligned}
C(\delta t) & =\frac{k_{B} T}{\left\{8 \pi v_{s} K|\delta t|+\frac{2 \pi K\left\langle\left[\Delta \mathbf{r}_{s}^{\perp}(\delta t)\right]^{2}\right\rangle}{\lambda}\right\}} \\
& \approx \frac{k_{B} T}{2 \pi \sqrt{B K}\left\langle\left[\Delta \mathbf{r}_{s}^{\perp}(\delta t)\right]^{2}\right\rangle},
\end{aligned}
$$

where in the last, approximate, equality, we have assumed (as we will verify a posteriori is true in the limit $\delta t \rightarrow 0$ ) that $\left\langle\left[\Delta \mathbf{r}_{s}^{\perp}(\delta t)\right]^{2}\right\rangle \gg \lambda v_{s}|\delta t|$. This assumption amounts to assuming that there is anomalous diffusion in this case, as we will now show.

Using the approximate equality of (28) in (10) leads to a self-consistent equation for $\left\langle\left[\Delta \mathbf{r}_{s}^{\perp}(t)\right]^{2}\right\rangle$ :

$$
\left\langle\left[\Delta \mathbf{r}_{s}^{\perp}(t)\right]^{2}\right\rangle=\frac{k_{B} T v_{s}^{2}}{2 \pi \sqrt{B K}} \int_{0}^{t} d t^{\prime} \int_{0}^{t} d t^{\prime \prime} \frac{1}{\left\langle\left[\Delta \mathbf{r}_{s}^{\perp}\left(t^{\prime}-t^{\prime \prime}\right)\right]^{2}\right\rangle} .
$$

We will seek a self-consistent solution to this equation of the form

$$
\left\langle\left[\Delta \mathbf{r}_{s}^{\perp}(t)\right]^{2}\right\rangle=\bar{D} t\left[\ln \left(\frac{t}{t_{0}}\right)\right]^{\alpha},
$$

where $t_{0}$ is a short-time cutoff, and $\alpha$ and $\bar{D}$ are, respectively, an exponent and a "superdiffusion" constant, both of which we will determine self-consistently. This leads to the condition

$$
\begin{aligned}
& \bar{D} t\left[\ln \left(\frac{t}{t_{0}}\right)\right]^{\alpha} \\
& =\frac{k_{B} T}{2 \pi \bar{D} \sqrt{B K}} \int_{0}^{t} d t^{\prime} \int_{0}^{t} d t^{\prime \prime} \frac{1}{\left|t^{\prime}-t^{\prime \prime}\right|\left[\ln \left(\frac{\left|t^{\prime}-t^{\prime \prime}\right|^{\prime}}{t_{0}}\right)\right]^{\alpha}} \\
& =\frac{k_{B} T}{2 \pi \bar{D} \sqrt{B K}(1-\alpha)} t\left[\ln \left(\frac{t}{t_{0}}\right)\right]^{1-\alpha},
\end{aligned}
$$

where in the second step the integrals over $t^{\prime}$ and $t^{\prime \prime}$ exclude the region in which $\left|t^{\prime}-t^{\prime \prime}\right|<t_{0}$. This expression (31) is clearly satisfied if $\alpha=1-\alpha$, so $\alpha=\frac{1}{2}$, and

$$
\bar{D}=\frac{v_{s}}{(B K)^{1 / 4}} \sqrt{\frac{k_{B} T}{\pi}} .
$$

Inserting these results into our ansatz (30) leads to our final prediction for asymptotic lateral diffusion of the swimmer at asymptotically large times:

$$
\left\langle\left[\Delta \mathbf{r}_{s}^{\perp}(t)\right]^{2}\right\rangle=\bar{D} t \sqrt{\ln \left(\frac{t}{t_{0}}\right)},
$$

with the anomalous diffusion constant $\bar{D}$ given by (32).

Recall that these results were derived on the assumption that the explicit dependence on $t^{\prime}-t^{\prime \prime}$ of $C_{\perp}$ in (14) could be neglected for sufficiently large times. In the next subsection, we 
will demonstrate that this is true both for the non-momentumconserving model that we simulate and for real smectics in which momentum is conserved. We will also estimate how large the time has to be before the asymptotic law (33) applies. This asymptotic time $t_{a}$ proves to be quite short for the non-momentum-conserving model but depends exponentially on parameters for momentum-conserving models. As a result, for many momentum-conserving models, $t_{a}$ is astronomically large, and a different, but still anomalous, scaling law, which we also derive below, applies.

\section{B. Asymptotic time for different dynamical models}

There are a number of different dynamical models for $u(\mathbf{r}, t)$ that will relax to the equilibrium distribution for a smectic A. We simulate a simple, purely relaxational model, which reduces at long wavelengths to

$$
\frac{\partial u(\mathbf{r}, t)}{\partial t}=-\Gamma \frac{\delta H}{\delta u}+f(\mathbf{r}, t),
$$

where the Hamiltonian $H$ is given by (19), and the noise $f$ in Eq. (34) must obey the fluctuation-dissipation theorem, which implies

$$
\left\langle f(\mathbf{r}, t) f\left(\mathbf{r}^{\prime}, t^{\prime}\right)\right\rangle=2 \Gamma k_{\mathrm{B}} T \delta^{3}\left(\mathbf{r}-\mathbf{r}^{\prime}\right) \delta\left(t-t^{\prime}\right) .
$$

These dynamics differ from those of real bulk smectics [40], which are complicated by the coupling of the layer displacement field $u(\mathbf{r}, t)$ to background fluid flow. In the important range of wave vectors $q_{z} \lesssim \lambda q_{\perp}^{2},|\mathbf{q}| \ll a^{-1}$ (where $a$ is the smectic layer spacing), these equations reduce to [40]

$$
\begin{gathered}
\frac{\partial u(\mathbf{r}, t)}{\partial t}=g_{z} / \rho_{0}, \\
\frac{\partial g_{z}(\mathbf{r}, t)}{\partial t}=-\frac{\delta H}{\delta u}+\frac{\eta_{2}}{\rho_{0}} \nabla_{\perp}^{2} g_{z}+f_{z}(\mathbf{r}, t),
\end{gathered}
$$

where $g_{z}$ is the local $z$ component of the momentum density of the smectic, $\rho_{0}$ is the mean density of the smectic, $\eta_{2}$ is one of the five viscosities characterizing the viscous response of uniaxial systems like smectics A, the elastic Hamiltonian $H$ is still given by (19), and the noise $f_{z}$ is constrained by the fluctuation-dissipation theorem to satisfy

$$
\left\langle f(\mathbf{r}, t) f\left(\mathbf{r}^{\prime}, t^{\prime}\right)\right\rangle=2 \eta_{2} k_{\mathrm{B}} T \nabla_{\perp}^{2} \delta^{3}\left(\mathbf{r}-\mathbf{r}^{\prime}\right) \delta\left(t-t^{\prime}\right),
$$

again in the wave-vector regime of interest.

We will now consider each of these models in turn and show that the long-time limit of the superdiffusive behavior is given by (33) for both of them. We will also calculate the asymptotic time $t_{a}$ for both models, and derive the alternative superdiffusive law for $t \ll t_{a}$ in the momentum-conserving case, for which $t_{a}$ can be astronomically large.

\section{Non-momentum-conserving model}

We seek the space and time-dependent correlation function $C_{\perp}(\mathbf{r}, t)$ in Eq. (14) of the director. As before, we will obtain this from the correlations of the displacement field $u(\mathbf{r}, t)$. These can readily be obtained from the equation of motion (34) by Fourier transforming in space, solving the resultant ordinary differential equation for $u(\mathbf{q}, t)$ in terms of $f_{z}(\mathbf{q}, t)$, and autocorrelating the result at two different times. This gives

$$
\langle u(\mathbf{q}, t+\tau) u(-\mathbf{q}, \tau)\rangle=\frac{k_{B} T e^{-\Gamma G_{\mathbf{q}} t}}{G_{\mathbf{q}}},
$$

where we have defined

$$
G_{\mathbf{q}} \equiv K q_{\perp}^{4}+B q_{z}^{2}
$$

This implies that director correlations in Fourier space are given by

$$
\left\langle\mathbf{n}_{\perp}(\mathbf{q}, t+\tau) \cdot \mathbf{n}_{\perp}(-\mathbf{q}, \tau)\right\rangle=\frac{k_{B} T q_{\perp}^{2} e^{-\Gamma G_{\mathbf{q}} t}}{G_{\mathbf{q}}},
$$

which in turn implies that real space director fluctuations are given by

$$
\begin{aligned}
C(\mathbf{r}, t) & =\left\langle\mathbf{n}_{\perp}(\mathbf{r}+\mathbf{R}, \tau+t) \cdot \mathbf{n}_{\perp}(\mathbf{R}, \tau)\right\rangle \\
& =k_{B} T \int \frac{d^{3} q}{8 \pi^{3}} \frac{q_{\perp}^{2} e^{i \mathbf{q} \cdot \mathbf{r}-\Gamma G_{\mathbf{q}} t}}{G_{\mathbf{q}}} .
\end{aligned}
$$

Changing variables of integration in this multiple integral from $\mathbf{q}_{\perp}$ to $\mathbf{Q}_{\perp} \equiv\left|\mathbf{r}_{\perp}\right| \mathbf{q}_{\perp}$ and from $q_{z}$ to $Q_{z} \equiv\left|\mathbf{r}_{\perp}\right|^{2} q_{z} / \lambda$ enables us to rewrite this in a scaling form:

$$
C(\mathbf{r}, t)=\frac{k_{B} T}{\sqrt{B K}\left|\mathbf{r}_{\perp}\right|^{2}} \Upsilon_{N}\left(\frac{\lambda z}{\left|\mathbf{r}_{\perp}\right|^{2}}, \frac{\Gamma K t}{\left|\mathbf{r}_{\perp}\right|^{4}}\right),
$$

where we have defined the scaling function

$\Upsilon_{N}(\psi, \zeta)=\int \frac{d^{3} Q}{8 \pi^{3}} \frac{Q_{\perp}^{2} \exp \left[i\left(Q_{x}+Q_{z} \psi\right)-\zeta\left(Q_{z}^{2}+Q_{\perp}^{4}\right)\right]}{Q_{z}^{2}+Q_{\perp}^{4}}$.

To justify the equal-time approximation made in the preceding section, we need to show that the explicit time dependence of this correlation function (43) can be neglected. From the scaling form, we see that this will be a good approximation whenever the dimensionless scaling variable $\zeta=\frac{\Gamma K t}{\left|\mathbf{r}_{\perp}\right|^{4}}$ associated with time is small; that is, $\zeta \ll 1$. Using our result (33) from the previous section for $\left\langle\left|\mathbf{r}_{\perp}\right|^{2}\right\rangle$ and estimating the typical value of $\left|\mathbf{r}_{\perp}\right|^{4} \sim\left\langle\left|\mathbf{r}_{\perp}\right|^{2}\right\rangle^{2}$ leads to the condition for the validity of our asymptotic result (33):

$$
\frac{\Gamma K}{\bar{D}^{2} t \ln \left(\frac{t}{t_{0}}\right)} \ll 1
$$

which is clearly always satisfied at long times. Indeed, it is satisfied whenever

$$
t \gg t_{a} \equiv \frac{\Gamma K}{\bar{D}^{2}}=\frac{\Gamma K \sqrt{B K}}{k_{\mathrm{B}} T v_{s}^{2}} .
$$

Since $t_{a}$ is a fairly weak function (i.e., algebraic, rather than exponential, as in the momentum-conserving case) of the parameters of our model, we expect it to be fairly easy to reach the asymptotic regime $t \gg t_{a}$, in which our asymptotic result (33) applies. And indeed, we find in our simulations that (33) holds from very early times out to the longest times we can simulate, as we have just predicted. 


\section{Momentum-conserving model}

In Fourier space, our momentum-conserving model becomes

$$
\begin{gathered}
\frac{\partial u(\mathbf{q}, t)}{\partial t}=g_{z}(\mathbf{q}, t) / \rho_{0}, \\
\frac{\partial g_{z}(\mathbf{q}, t)}{\partial t}=-G_{\mathbf{q}} u-\frac{\eta_{2}}{\rho_{0}} q_{\perp}^{2} g_{z}(\mathbf{q}, t)+f_{z}(\mathbf{q}, t),
\end{gathered}
$$

where $G_{\mathbf{q}}$ was defined in (40). If we assume we are in the Stokesian limit, in which the viscous $\left(\eta_{2}\right)$ term dominates the inertial $\frac{\partial g_{z}(\mathbf{q}, t)}{\partial t}$ term in (48), then we can solve that equation directly for $g_{z}(\mathbf{q}, t)$, obtaining

$$
g_{z}(\mathbf{q}, t)=-\rho_{0}\left[\frac{G_{\mathbf{q}} u(\mathbf{q}, t)-f_{z}(\mathbf{q}, t)}{\eta_{2} q_{\perp}^{2}}\right] .
$$

Inserting this into the equation of motion (47) for $u(\mathbf{q}, t)$ gives

$$
\frac{\partial u(\mathbf{q}, t)}{\partial t}=-\left[\frac{G_{\mathbf{q}} u(\mathbf{q}, t)-f_{z}(\mathbf{q}, t)}{\eta_{2} q_{\perp}^{2}}\right] .
$$

We can now check a posteriori our assumption that we are in the Stokesian limit by using this expression to compute $\frac{\partial g_{z}(\mathbf{q}, t)}{\partial t}$ and taking its ratio with the $\eta_{2}$ term in (48). Doing so, and keeping only the $u$-dependent terms, we find this ratio is $R \equiv$ $\frac{\partial_{t} g_{z}(\mathbf{q}, t)}{\frac{\eta_{2}}{\rho_{0}} q_{\perp}^{2} g_{z}} \sim \frac{\rho_{0} G_{\mathbf{q}}}{\eta_{2}^{2} q_{\perp}^{4}}$. This ratio is clearly a monotonically increasing function of $q_{z}^{2}$, so it is biggest when $q_{z}=0$, at which point it is given by $\frac{K \rho_{0}}{\eta_{2}^{2}} \equiv \chi$. The smaller this ratio, the better our Stokesian approximation. For typical thermotropic smectics, $K \sim 5 \times 10^{-7}$ dynes, $\rho_{0} \sim 1 \frac{\text { gram }}{\mathrm{cm}-\mathrm{sec}}$, and $\eta_{2} \sim 1$ poise, which gives $\chi \sim 5 \times 10^{-7}$. Even in lyotropic smectics, for which $\eta_{2}$ approaches the viscosity of water, which is two orders of magnitude smaller than the value of $\eta_{2}$ we have just used, we still get $\chi \sim 5 \times 10^{-3}$, and this is before taking into account the reduction of $K$ due to dilution. So our Stokesian approximation is clearly a very good one in all cases.

The correlation functions of $u$ can now be obtained from (50) as in the nonconserving case, by Fourier transforming in space, solving the resultant ordinary differential equation for $u(\mathbf{q}, t)$ in terms of $f_{z}(\mathbf{q}, t)$, and autocorrelating the result at two different times. This gives

$$
\langle u(\mathbf{q}, t+\tau) u(-\mathbf{q}, \tau)\rangle=\frac{k_{B} T \exp \left(-\frac{G_{\mathbf{q}}}{\eta_{2} q_{\perp}^{2}} t\right)}{G_{\mathbf{q}}} .
$$

This implies that director correlations in Fourier space are given by

$$
\left\langle\mathbf{n}_{\perp}(\mathbf{q}, t+\tau) \cdot \mathbf{n}_{\perp}(-\mathbf{q}, \tau)\right\rangle=\frac{k_{B} T q_{\perp}^{2} \exp \left(-\frac{G_{\mathbf{q}}}{\eta_{2} q_{\perp}^{2}} t\right)}{G_{\mathbf{q}}},
$$

which in turn implies that real space director fluctuations are given by

$$
\begin{aligned}
C(\mathbf{r}, t) & =\left\langle\mathbf{n}_{\perp}(\mathbf{r}+\mathbf{R}, \tau+t) \cdot \mathbf{n}_{\perp}(\mathbf{R}, \tau)\right\rangle \\
& =k_{B} T \int \frac{d^{3} q}{8 \pi^{3}} \frac{q_{\perp}^{2} e^{i \mathbf{q} \cdot \mathbf{r}-\frac{G_{\mathbf{q}}}{\eta_{2} q_{\perp}^{2}}}}{G_{\mathbf{q}}} .
\end{aligned}
$$

Changing variables of integration in this multiple integral from $\mathbf{q}_{\perp}$ to $\mathbf{Q}_{\perp} \equiv\left|\mathbf{r}_{\perp}\right| \mathbf{q}_{\perp}$ and from $q_{z}$ to $Q_{z} \equiv\left|\mathbf{r}_{\perp}\right|^{2} q_{z} / \lambda$ enables us to rewrite this in a scaling form:

$$
C(\mathbf{r}, t)=\frac{k_{B} T}{\sqrt{B K}\left|\mathbf{r}_{\perp}\right|^{2}} \Upsilon_{C}\left(\frac{\lambda z}{\left|\mathbf{r}_{\perp}\right|^{2}}, \frac{K t}{\eta_{2}\left|\mathbf{r}_{\perp}\right|^{2}}\right),
$$

where we have defined the scaling function

$\Upsilon_{C}(\psi, \zeta)=\int \frac{d^{3} Q}{8 \pi^{3}} \frac{Q_{\perp}^{2} \exp \left[i\left(Q_{x}+Q_{z} \psi\right)-\zeta\left(\frac{Q_{z}^{2}}{Q_{\perp}^{2}}+Q_{\perp}^{4}\right)\right]}{Q_{z}^{2}+Q_{\perp}^{4}}$.

To justify the equal-time approximation made in the preceding section, we need to show that the explicit time dependence of this correlation function (43) can be neglected. From the scaling form, we see that this will be a good approximation whenever the dimensionless scaling variable $\zeta=\frac{K t}{\eta_{2}\left|\mathbf{r}_{\perp}\right|^{2}}$ associated with time is small; that is, $\zeta \ll 1$. Using our result (33) from the previous section for $\left\langle\left|\mathbf{r}_{\perp}\right|^{2}\right\rangle$, and estimating the typical value of $\left|\mathbf{r}_{\perp}\right|^{2} \sim\left\langle\left|\mathbf{r}_{\perp}\right|^{2}\right\rangle$ leads to the condition for the validity of our asymptotic result (33):

$$
\frac{K t}{\eta_{2} \bar{D} t \sqrt{\ln \left(\frac{t}{t_{0}}\right)}} \ll 1,
$$

which is clearly always satisfied at long times. Indeed, it is satisfied whenever

$$
t \gg t_{a} \equiv t_{0} \exp \left[\left(\frac{K}{\eta_{2} \bar{D}}\right)^{2}\right] \equiv t_{0} \exp \left[\left(\frac{v_{c}}{v_{s}}\right)^{2}\right],
$$

where we have defined a characteristic velocity

$$
v_{c} \equiv \frac{B^{1 / 4} K^{5 / 4}}{\eta_{2}} \sqrt{\frac{\pi}{k_{\mathrm{B}} T}} .
$$

In deriving this expression, we have used Eq. (32) for $\bar{D}$.

We see from Eq. (57) that, in contrast to the non-momentumconserving case, when momentum is conserved, the asymptotic time (57) is extremely sensitive (indeed, exponentially so) to material parameters and to the speed of the swimmer. It can also become astronomically large. Taking typical numbers for a thermotropic smectic, such as $B \sim 5 \times 10^{7} \frac{\text { dynes }}{\mathrm{cm}^{2}}, K \sim$ $5 \times 10^{-7}$ dynes, and $\eta_{2} \sim 1$ poise gives $v_{c} \sim 5 \frac{\mathrm{cm}}{\mathrm{sec}}$. Putting a bacterium with a swimming speed of $v_{s} \sim 50 \frac{\mu}{\mathrm{sec}}$ in such a smectic, we see that Eq. (57) implies an asymptotic time of $t_{a}=t_{0} \exp \left(10^{4}\right)$, which, for any reasonable $t_{0}$, is far longer than the age of the universe!

However, the extreme exponential sensitivity of the asymptotic time $t_{a}$ means that it should be achievable in other systems. Lyotropic smectics are a good candidate. In a highly dilute lyotropic smectic, the elastic constants obey [41] $B \sim \frac{\left(k_{\mathrm{B}} T\right)^{2}}{\kappa \ell^{3}}$, and $K \sim \frac{\kappa}{\ell}$, where $\ell$ is the lamellar spacing and $\kappa$ is the bend rigidity per unit area of a single lamella. Inserting these expressions into our expression (58) gives

$$
v_{c} \sim \frac{\kappa}{\eta_{2} \ell^{2}} .
$$

Thus, for very dilute systems, in which $\ell$ is large, we can make the characteristic speed $v_{c}$ very small. Taking a typical lamellar bend stiffness $\kappa \sim 5 \times 10^{-14}$ ergs and noting that for a highly dilute lamellar phase, we expect and $\eta_{2} \sim \eta_{\mathrm{H}_{2} \mathrm{O}} \sim 10^{-2}$ poise, 
we obtain from (59)

$$
v_{c} \sim 5 \frac{\mu}{\sec }\left(\frac{1 \mu}{\ell}\right)^{2},
$$

which implies from (57) that for a lamellar phase with a layer spacing of $\ell=0.3 \mu \mathrm{m}$, and a bacteria swimming at $v_{s}=50 \frac{\mu}{\mathrm{sec}}$, the asymptotic time would be

$$
t_{a} \sim t_{0} e^{(10 / 9)^{2}} \approx 3.44 t_{0},
$$

which should be quite experimentally accessible.

What about those cases in which $t_{a}$ is astronomically large? We can show that in those cases, there is also anomalous diffusion, but with a different scaling law. To see this, we note that $t \ll t_{a}$, the time variable is now the dominant one in the scaling law (54) for the correlation function. Hence, we can evaluate that correlation function setting $\mathbf{r}_{\perp}$ and $z$ to zero in our general expression (53) for $C_{\perp}$. This gives

$$
\begin{aligned}
C(\mathbf{r}=\mathbf{0}, t) & =\left\langle\mathbf{n}_{\perp}(\mathbf{R}, \tau+t) \cdot \mathbf{n}_{\perp}(\mathbf{R}, \tau)\right\rangle \\
& =k_{B} T \int \frac{d^{3} q}{8 \pi^{3}} \frac{q_{\perp}^{2} e^{-\frac{G_{\mathbf{q}}}{\eta_{2} q_{\perp}^{2}}}}{G_{\mathbf{q}}} .
\end{aligned}
$$

With the change of variables $\mathbf{q}_{\perp}$ to $\mathbf{Q}_{\perp} \equiv \sqrt{\frac{K t}{\eta_{2}}} \mathbf{q}_{\perp}$ and from $q_{z}$ to $Q_{z} \equiv q_{z} \frac{K t}{\eta_{2} \lambda}$, we can pull the time dependence out of this expression, obtaining

$$
C(\mathbf{r}=\mathbf{0}, t)=\left(\frac{k_{\mathrm{B}} T \eta_{2}}{8 \pi^{3} \sqrt{B K^{3}}}\right) \frac{f(1)}{t},
$$

where we have defined

$$
f(x) \equiv \int d^{3} Q \frac{Q_{\perp}^{2} e^{-x\left(\frac{Q_{z}^{2}+Q_{\perp}^{4}}{Q_{\perp}^{2}}\right)}}{Q_{z}^{2}+Q_{\perp}^{4}} .
$$

This function can easily be evaluated by differentiating it with respect to $x$; this gives

$$
f^{\prime}(x)=\int d^{3} Q e^{-x \frac{Q_{z}^{2}+Q_{\perp}^{4}}{Q_{\perp}^{2}}} .
$$

Performing the Gaussian integral over $Q_{z}$ gives

$$
f^{\prime}(x)=\int d^{2} Q_{\perp} e^{-x Q_{\perp}^{2}} Q_{\perp} \sqrt{\frac{\pi}{x}} .
$$

The integral $d^{2} Q_{\perp}$ is also elementary; we thereby obtain

$$
f^{\prime}(x)=-\frac{\pi^{2}}{2 x^{2}}
$$

Integrating this, and determining the unknown constant of integration by noting, from inspection of (65), that $f(x \rightarrow$ $\infty) \rightarrow 0$, we obtain

$$
f(x)=\frac{\pi^{2}}{2 x} .
$$

Using this in (63) gives

$$
C(\mathbf{r}=\mathbf{0}, t)=\left(\frac{k_{\mathrm{B}} T \eta_{2}}{16 \pi \sqrt{B K^{3}}}\right) \frac{1}{t} .
$$

Now replacing $\left\langle\mathbf{n}_{\perp}\left(\mathbf{r}_{s}\left(t^{\prime}\right), t^{\prime}\right) \cdot \mathbf{n}_{\perp}\left(\mathbf{r}_{s}\left(t^{\prime \prime}\right), t^{\prime \prime}\right)\right\rangle$ in Eq. (10) with $C\left(\mathbf{r}=\mathbf{0}, t^{\prime}-t^{\prime \prime}\right)$ from this expression, and doing the $t^{\prime}$ and $t^{\prime \prime}$ integrals in (10) gives, again, anomalous diffusion, but with a different scaling law:

$$
\left\langle\left[\Delta \mathbf{r}_{s}^{\perp}(t)\right]^{2}\right\rangle=\left(\frac{k_{\mathrm{B}} T \eta_{2}}{16 \pi \sqrt{B K^{3}}}\right) t \ln \left(\frac{t}{t_{0}}\right) .
$$

This is the expression that is most experimentally relevant to thermotropic smectics, or to lyotropics at lower dilutions (i.e., lamellar spacings $\ell \ll 1 \mu$ ).

\section{SIMULATION MODEL}

We will now attempt to corroborate the theoretical predictions for the long-time swimmer dynamics in the smectic fluid using a particle-based simulation model. The model potential employed in our simulations is designed to generate stable nematic and smectic (A) phases at low temperature while producing trivial isotropic fluids at high temperature. It corresponds to a simple soft-core potential proposed in Ref. [42] and models the interaction energy $U_{s c}$ between two soft spherocylinders at center-of-mass displacement $\Delta \mathbf{r}$ with orientation unit vectors $\hat{\mathbf{u}}$ and $\hat{\mathbf{u}}^{\prime}$ :

$$
U_{s c}= \begin{cases}u_{m}(1-\sigma)^{2}+\epsilon, & \sigma<1 \\ u_{m}(1-\sigma)^{2}-U_{a}(1-\sigma)^{4}+\epsilon, & 1 \leqslant \sigma<\sigma_{c}, \\ 0 & \sigma \geqslant \sigma_{c}\end{cases}
$$

where $\sigma\left(\Delta \mathbf{r}, \hat{\mathbf{u}}, \hat{\mathbf{u}}^{\prime}\right)$ denotes the shortest distance between two short spherocylinders of length $L$ and diameter $\sigma_{0}$ at fixed mutual orientation. The attractive part of the potential takes the form

$$
U_{a}=u_{a}-5 \epsilon_{1} \mathcal{P}_{2}\left(\hat{\mathbf{u}} \cdot \hat{\mathbf{u}}^{\prime}\right)-5 \epsilon_{2}\left[\mathcal{P}_{2}(\Delta \hat{\mathbf{r}} \cdot \hat{\mathbf{u}})+\mathcal{P}_{2}\left(\Delta \hat{\mathbf{r}} \cdot \hat{\mathbf{u}}^{\prime}\right)\right]
$$

in terms of a second-order Legendre polynomial $\mathcal{P}_{2}$ and centerof-mass distance unit vector $\Delta \hat{\mathbf{r}}$. Furthermore, $\epsilon\left(\Delta \hat{\mathbf{r}}, \hat{\mathbf{u}}, \hat{\mathbf{u}}^{\prime}\right)=$ $-u_{m}^{2} / 4 U_{a}\left(\Delta \hat{\mathbf{r}}, \hat{\mathbf{u}}, \hat{\mathbf{u}}^{\prime}\right)$ is the maximum well depth for the configuration chosen such as to guarantee the potential and its first derivative to reach zero at the cutoff distance $\sigma_{c}\left(\Delta \hat{\mathbf{r}}, \hat{\mathbf{u}}, \hat{\mathbf{u}}^{\prime}\right)=$ $1+\sqrt{u_{m} / 2 U_{a}\left(\Delta \hat{\mathbf{r}}, \hat{\mathbf{u}}, \hat{\mathbf{u}}^{\prime}\right)}$. The shape of the soft-core potential can be judiciously tuned through the four-parameter combination $\left\{u_{m}, u_{a}, \epsilon_{1}, \epsilon_{2}\right\}$ enabling facile simulation of a range of liquid crystalline mesophases [42].

The swimmer is described as a point particle with position $\mathbf{r}_{s}$ and orientation unit vector $\hat{\mathbf{u}}_{s}$. It interacts with the surrounding soft rods by means of a coupling potential [23] of strength $\epsilon_{s}$ :

$$
U_{s}=\epsilon_{s} \sum_{i} \mathcal{P}_{2}\left(\hat{\mathbf{u}}_{i} \cdot \hat{\mathbf{u}}_{s}\right) g\left(r_{i, s}\right) .
$$

The coupling potential decays with increasing distance $r_{i, s}=$ $\left\|\mathbf{r}_{i, s}\right\|$ between the swimmer and the soft rods through a Gaussian $g\left(r_{i, s}\right)=\exp \left[-\left(r_{i, s} / \sigma_{s}\right)^{2}\right]$ with $\sigma_{s}$ a characteristic length scale setting the coupling range.

The microscopic equations of motion for the positional coordinates describe overdamped Brownian motion of each $\operatorname{rod} i$ :

$$
\xi \cdot \partial_{t} \mathbf{r}_{i}=-\nabla_{\mathbf{r}_{i}} U\left(\left\{\mathbf{r}_{i}, \hat{\mathbf{u}}_{i}\right\}\right)+\overline{\mathbf{f}}_{i},
$$

where $\xi=\xi^{\|} \hat{\mathbf{u}} \hat{\mathbf{u}}+\xi^{\perp}(\mathbf{I}-\hat{\mathbf{u}} \hat{\mathbf{u}})$ denotes the translational friction tensor of a uniaxial rod. The first term on the rhs is a direct force on rod $i$ due to presence of neighboring liquid crystal particles via the total potential energy $U=\frac{1}{2} \sum_{i, j} U_{s c}$, assumed pairwise additive. Furthermore, $\bar{f}^{\alpha}=\sqrt{2 \xi_{\alpha} k_{B} T} R_{\alpha}(t)$ 
is a Gaussian random force acting on each rod with zero mean $\left\langle R_{\alpha}(t)\right\rangle=0$ and variance $\left\langle R_{\alpha}(t) R_{\gamma}\left(t^{\prime}\right)\right\rangle=\delta_{\alpha \gamma} \delta\left(t-t^{\prime}\right)$ with $\alpha, \gamma$ indicating the components of a rod-based orthonormal frame $\overline{\mathbf{f}}=\bar{f}^{\|} \hat{\mathbf{u}}+\bar{f}^{\perp 1} \hat{\mathbf{e}}_{1}+\bar{f}^{\perp 2} \hat{\mathbf{e}}_{2}$. The equation of motion for the orientation of the soft-core particles follows from a similar balance of torques, via

$$
\xi_{R} \partial_{t} \hat{\mathbf{u}}_{i}=\left(\mathbf{w}_{i}+\mathbf{w}_{i, s}+\overline{\mathbf{w}}\right) \times \hat{\mathbf{u}}_{i},
$$

with $\xi_{R}$ the rotational friction factor, $\mathbf{w}_{i}=\lambda_{i} \hat{\mathbf{u}}_{i}-\partial U / \partial \hat{\mathbf{u}}_{i}$ the torque on particle $i$ due to the surrounding rods (with $\lambda_{i}$ a Lagrange multiplier ensuring normalization of $\left.\hat{\mathbf{u}}_{i}\right), \mathbf{w}_{i, s}=$ $\lambda_{i} \hat{\mathbf{u}}_{i}-\partial U_{s} / \partial \hat{\mathbf{u}}_{i}$ the contribution imparted by the presence of the swimmer, and $\bar{\omega}_{\alpha}=\sqrt{2 \xi_{R} k_{B} T} R_{\alpha}(t)$ a random Gaussian torque within the orthonormal particle frame so that $\alpha=\{\perp 1, \perp 2\}$. The geometric factors $\left\{\xi_{\|}, \xi_{\perp}, \xi_{R}\right\}$ depend solely on the rod aspect ratio $p=\left(L+\sigma_{0}\right) / \sigma_{0}>0$, and we adopt the standard expressions for rodlike macromolecules, as given in Ref. [43]:

$$
\begin{aligned}
\frac{\xi_{0}}{\xi_{\perp}} & =\frac{1}{4 \pi}\left(\ln p+0.839+0.185 / p+0.233 / p^{2}\right), \\
\frac{\xi_{0}}{\xi_{\|}} & =\frac{1}{2 \pi}\left(\ln p-0.207+0.980 / p-0.133 / p^{2}\right), \\
\frac{\xi_{R 0}}{\xi_{R}} & =\frac{3}{2 \pi p^{2}}\left(\ln p-0.207+0.980 / p-0.133 / p^{2}\right)
\end{aligned}
$$

with $\xi_{0}$ and $\xi_{R 0}$ the friction factors of a reference sphere with radius $\sigma_{0}$. Defining $D_{0}=k_{B} T / \xi_{0}$ as the translational diffusion coefficient of a reference sphere.

The trajectory $\left\{\mathbf{r}_{s}(t), \hat{\mathbf{u}}_{s}(t)\right\}$ of the swimmer is governed by the following equations of motion:

$$
\begin{aligned}
& \xi_{s} \cdot \partial_{t} \mathbf{r}_{s}=f_{a} \hat{\mathbf{u}}_{s}, \\
& \xi_{R s} \partial_{t} \hat{\mathbf{u}}_{s}=\left(\mathbf{w}_{s, i}+\overline{\mathbf{w}}_{s}\right) \times \hat{\mathbf{u}}_{s},
\end{aligned}
$$

with $\xi_{R s}$ the rotational friction factor and $\xi_{s}$ the translational friction tensor of the swimmer, $\mathbf{w}_{s, i}=\lambda_{s} \hat{\mathbf{u}}_{s}-\partial U_{s} / \partial \hat{\mathbf{u}}_{s}$ the torque acting on the swimmer imparted by neighboring soft rods and $\overline{\mathbf{w}}_{s}$ a random torque. The geometric factors are identical to Eq. (76) for a given hydrodynamic swimmer aspect ratio $p_{s}>1$ which may be different from that of the soft rods $p$. The translational noise on the swimmer is ignored as it is assumed to be negligible compared to the orientational noise (as is the case for motile bacteria [44]). Throughout this study, we use dimensionless expressions for time $\tau=t D_{0} / \sigma_{0}^{2}$, temperature $T^{*}=k_{B} T / u_{a}$, particle density $\rho^{*}=N \sigma_{0}^{3} / V$ (with $N$ the number of particles and $V$ the system volume), and active force $f_{a}^{*}=f_{a} \sigma_{0} / \epsilon_{s}$.

The interaction parameters (in units $k_{B} T$ ) for the current model are $u_{m}=25, u_{a}=150, \epsilon_{1}=10$, and $\epsilon_{2}=-2$. The rod aspect ratio is fixed at $p=L / \sigma_{0}=3.0$. For these values the system undergoes a transformation from an isotropic, to a nematic and a smectic phase upon lowering the temperature and/or increasing particle density. We employ a cubic simulation box with periodic boundary conditions in all three directions. The initial configuration represents $N=3500$ rods forming a square smectic lattice at a given density $\rho^{*}$. It is allowed to melt during an equilibration run of at least $\Delta \tau=$ 2000. Once the host phase is equilibrated, a swimmer is placed in the center of the simulation box, and the whole system is reequilibrated during at least $\Delta \tau=2000$. To avoid numerical artifacts the time step associated with the linear discretization of the equations of motion is kept sufficiently small $(\delta \tau<$ 0.001). The parameters defining the interaction between the swimmer and the host rods are $\epsilon_{s}=1 k_{B} T, \sigma_{s}=\left(L+\sigma_{0}\right) / 2$ for the coupling strength and range, respectively, and $p_{s}=5$ for the swimmer aspect ratio. Production runs during which observables of interest were recorded span a total time interval of at least $\Delta \tau=5000$.

Let us define the displacement vectors $\Delta \mathbf{r}_{s}^{\|}(\tau) \equiv\left\{\left[\mathbf{r}_{s}(\tau)-\right.\right.$ $\left.\left.\mathbf{r}_{s}(0)\right] \cdot \hat{\mathbf{n}}\right\} \hat{\mathbf{n}}$ along, and $\Delta \mathbf{r}_{s}^{\perp}(\tau) \equiv \Delta \mathbf{r}_{s}(\tau)-\Delta \mathbf{r}_{s}^{\|}(\tau)$ perpendicular to, the layer normal $\hat{\mathbf{n}}$, with $\Delta \mathbf{r}_{s}(\tau)=\mathbf{r}_{s}(\tau)-\mathbf{r}_{s}(0)$. We then determined from our simulations the mean squared displacements $\left\langle\left[\Delta \mathbf{r}_{s}^{\|}(\tau)\right]^{2}\right\rangle$ and $\left\langle\left(\Delta \mathbf{r}_{s}^{\perp}(\tau)\right)^{2}\right\rangle$, where $\langle\cdots\rangle$ denotes a time average in the steady state. We also monitor the mean-squared rotation of the swimmer via

$$
\left\langle\mathcal{P}_{n}\left[\hat{\mathbf{u}}_{s}(\tau) \cdot \hat{\mathbf{u}}_{s}(0)\right]\right\rangle, \quad n=1,2 .
$$

These quantities enable us to gauge the typical rotational relaxation time of the swimmer in relation to the symmetry of the medium and temperature. Since the effective shape of the swimmer is strongly anisotropic, i.e., $p_{s}>1$, the rate of hairpin turns is extremely small [23], and no such events are recorded during the course of our simulations except, of course, in the case of an isotropic background medium (at $T^{*} \gg 1$ ) where the orientational fluctuations of the swimmer are strong and the long-time dynamics becomes strictly diffusive.

An overview of the transverse MSDs for a swimmer moving is shown in Fig. 1(a). In this particular representation, the logarithmic long-time tails clearly show up in a linear fashion with the scaling exponent for the smectic systems differing significantly from those of the nematic systems. Note that, in Fig. 1(a), time is represented on an extremely compressed $\ln (\ln \tau)$ scale, and deviations from linear scaling occur at very long times where the statistics is no longer fully reliable. Running systematically longer simulation runs will obviously remedy this. The fitted exponents ( $\alpha=0.5 \pm 0.1$ for the smectic and $\alpha=1.0 \pm 0.1$ for the nematic) are in agreement with the ones established from our theoretical model. As expected, the mean-squared displacement parallel to the director remains ballistic throughout the sampled time interval for the nematic and smectic phases (suggesting hairpin turns to be completely absent), but for the isotropic phase it crosses over from ballistic to diffusive $\propto \tau$ beyond the typical effective rotation time of the swimmer. In general, for anisotropic systems such as nematic or smectic liquid crystals, the periodic boundary conditions impart a weak bias on the director and enforce it to align along one of the Cartesian axes of the simulation box or along the box diagonal. The effect of this bias can be systematically weakened by increasing the system size. We find, however, that the latter has no measurable impact on the long-time scaling of the transverse MSD, implying that the simulation setup does not break the rotational invariance of the nematic or smectic fluid.

The distinct lamellar signature of the smectic phase can be probed through the self-part of the van Hove function:

$$
G\left(z_{\|}, \tau\right)=\frac{1}{N}\left\langle\sum_{i=1}^{N} \int d r_{\|} \delta\left[z_{\|}-\left|\Delta \mathbf{r}_{i}^{\|}(\tau)\right|\right]\right\rangle,
$$

representing the average distribution of displacements of the host particles along the layer normal over time. The results, 

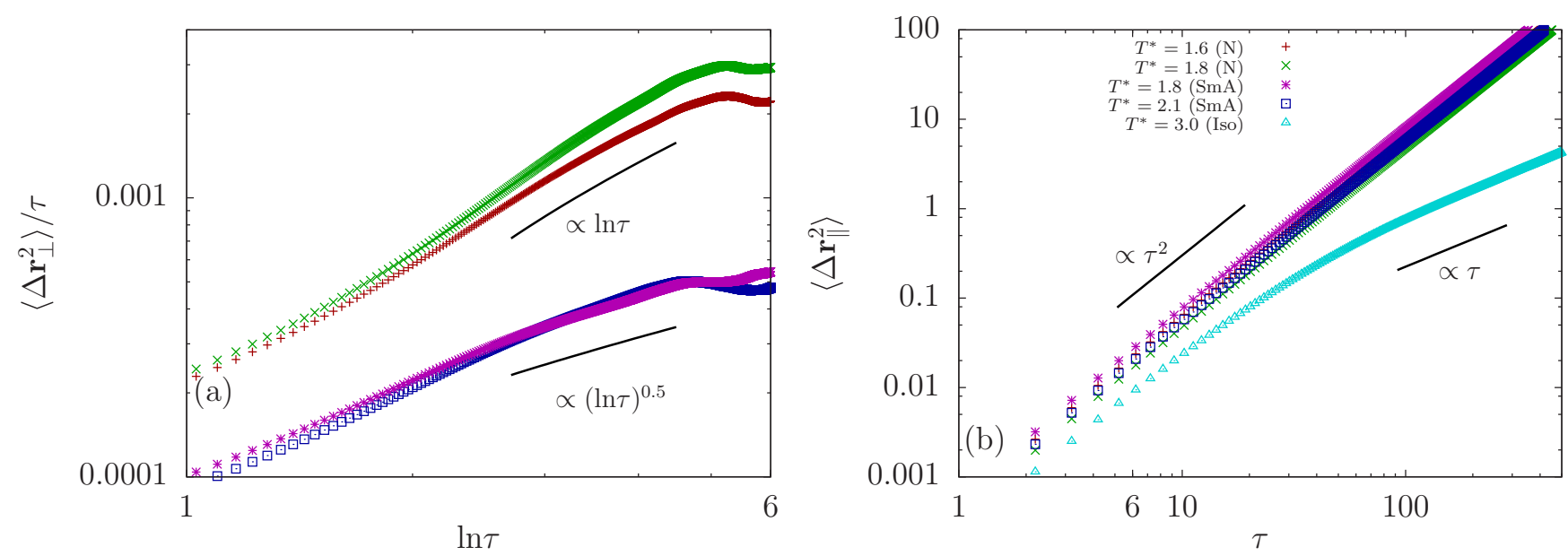

FIG. 1. MSDs of a swimmer moving through a nematic and smectic medium. Shown is the contribution (a) perpendicular and (b) parallel to the layer normal at various temperatures $T^{*}$. The rod densities corresponding to the various phases are $\rho^{*}=0.22$ (Iso), $\rho^{*}=0.3$ ( $\mathrm{N}$ ), and $\rho^{*}=0.5(\mathrm{SmA})$. The long-time dynamics transverse to the director is anomalous and exhibits a distinct long-time logarithmic behavior with scaling exponent $\alpha \approx 1$ for the nematic and $\alpha \approx 0.5$ for the smectic phase. For the isotropic phase, standard long-time swimmer diffusion $\left\langle\Delta \mathbf{r}^{2}\right\rangle \propto \tau$ is observed, as it should.

depicted in Fig. 2, enable a clear distinction between the Gaussian patterns of a spatially uniform nematic fluid, whereas the shoulders point to a lamellar microstructure of the smectic host phase.

In our simulations we have also tested an alternative model in which a swimmer is conceived simply by rendering one of the passive host rods active by applying an active force $f_{a}$ along its main orientation axis. In this model, the coupling between the swimmer and the host rods proceeds via the short-ranged, direct interaction given by Eq. (71). It turns out that both point- $\mathcal{P}_{2}$-type swimmer and rod-based swimmer produce the same asymptotic scaling of the transverse (and parallel) MSDs. This illustrates that the long-time scaling of the swimmer superdiffusion does not depend on the details of the swimmer-host coupling provided its propulsion direction is aligned along the local nematic director. A clear advantage of the rod-based swimmer is that it enables us to unambiguously recover standard long-time diffusion in an isotropic fluid [see Fig. 1(b)], while the mean-field $\mathcal{P}_{2}$ coupling interaction Eq. (73) tends to yield spurious results for isotropic systems. The latter is due to the fact that the number of host rods residing within a coupling range $\sigma_{s}$ of the swimmer centerof-mass is too limited in dilute environments to generate the correct orientational fluctuations on the swimmer, unless $\sigma_{s}$ is increased to impractically large values.

\section{CONCLUSIONS}

In this paper we have addressed the long-term motility of a swimmer dispersed in a smectic A (or lamellar) fluid phase. Using hydrodynamic theory for the swimmer dynamics subject to a fluctuating smectic director field, we have
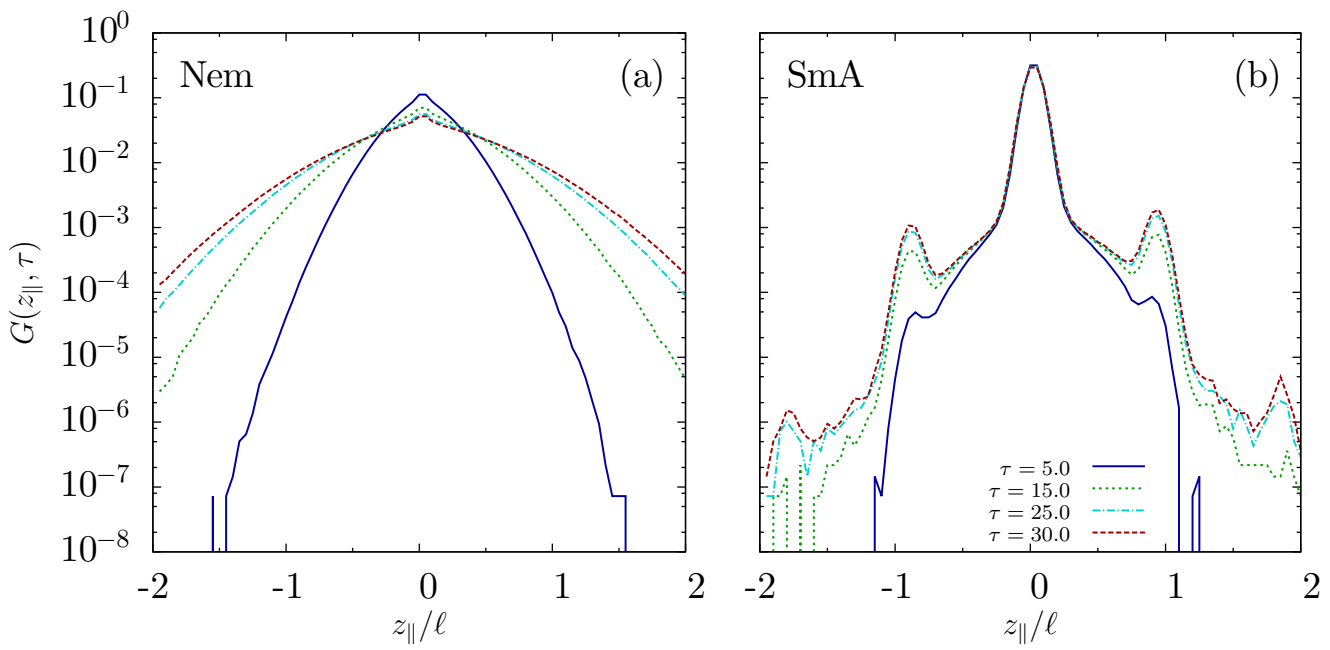

FIG. 2. Van Hove correlation function $G\left(z_{\|}, \tau\right)$ for the host rods forming a (a) nematic phase $\left(T^{*}=1.8, \rho^{*}=0.3\right)$ and (b) smectic phase $\left(T^{*}=1.8, \rho^{*}=0.5\right)$. Curves are depicted at various time $\tau$. The distinct shoulders at discrete rod lengths $\ell$ indicate the diffusive barriers imparted by the lamellar microstructure of the smectic phase. 
derived universal scaling expressions for the mean-squared swimmer displacement perpendicular to the director. The predictions are relevant to a vast range of smectic phases of both thermotropic and lyotropic origin and are independent of the dynamical process through which the nematic director field relaxes towards equilibrium. We find that the long-time lateral diffusion of the swimmer across the smectic membranes exhibits a logarithmic $\left[\propto t \sqrt{\ln \left(t / t_{0}\right)}\right]$ scaling with time $t$ which is distinctly different from the anomalous swimmer diffusion $\left[\propto t \ln \left(t / t_{0}\right)\right]$ in nematic fluids [23] or the trivial long-time diffusion $(\propto t)$ encountered in isotropic media. We corroborate our predictions using particle-based simulation of an active point particle moving through a smectic A phase composed of soft mesogens. Upon increasing temperature (and reducing particle concentration), the smectic host transforms into a nematic and, subsequently, an isotropic fluid. The concomitant transversal mean-squared swimmer displacement is fundamentally different in each of these phases, and the measured long-time scaling laws are in full agreement with theory. Given the universality of our theoretical predictions one could envisage as a possible experimental application measuring the long-time diffusion of swimming micro-organisms or active colloids propelling though liquid-crystalline media as a dynamical probe to identifying the microstructure of the anisotropic host phase. Efforts to relate our theoretical findings to experimental model systems of external field-controlled active colloids moving through thermotropic liquid crystals $[27,45]$ are currently being undertaken.

\section{ACKNOWLEDGMENTS}

We thank J. Ignés-Mullol and F. Sagués for helpful discussions. C.F.C. and H.H.W. gratefully acknowledge funding from the French National Research Agency (ANR) through the ANR-JCJC Grant "UPSCALE." H.L. was supported by the DFG within Project LO 418/20-1. J.T. thanks the Institut für Theoretische Physik II: Weiche Materie, Heinrich-HeineUniversität, Düsseldorf; the Max Planck Institute for the Physics of Complex Systems Dresden; the Department of Bioengineering at Imperial College, London; the Higgs Centre for Theoretical Physics at the University of Edinburgh; and the Lorentz Center of Leiden University for their hospitality while this work was underway.
[1] M. C. Marchetti, J. F. Joanny, S. Ramaswamy, T. B. Liverpool, J. Prost, M. Rao, and R. A. Simha, Rev. Mod. Phys. 85, 1143 (2013).

[2] P. Romanczuk, M. Bär, W. Ebeling, B. Lindner, and L. Schimansky-Geier, Eur. Phys. J. Spec. Top. 202, 1 (2012).

[3] J. Elgeti, R. G. Winkler, and G. Gompper, Rep. Prog. Phys. 78, 056601 (2015).

[4] C. Bechinger, R. Di Leonardo, H. Löwen, C. Reichhardt, G. Volpe, and G. Volpe, Rev. Mod. Phys. 88, 045006 (2016).

[5] M. F. Hagan and A. Baskaran, Curr. Opin. Cell Biol. 38, 74 (2016).

[6] M. A. Sleigh, J. R. Blake, and N. Liron, Am. Rev. Respir. Dis. 137, 726 (1988).

[7] S. S. Suarez and A. A. Pacey, Hum. Reprod. Update 12, 23 (2006).

[8] C. Josenhans and S. Suerbaum, Int. J. Med. Microbiol. 291, 605 (2002).

[9] H. R. Wallace, Annu. Rev. Phytopathol. 6, 91 (1968).

[10] H. C. Fu, T. R. Powers, and C. W. Wolgemuth, Phys. Rev. Lett. 99, 258101 (2007).

[11] X. N. Shen and P. E. Arratia, Phys. Rev. Lett. 106, 208101 (2011).

[12] D. A. Gagnon, N. C. Keim, and P. E. Arratia, J. Fluid Mech. 758, R3 (2014).

[13] B. Liu, T. R. Powers, and K. S. Breuer, Proc. Natl. Acad. Sci. USA 108, 19516 (2011).

[14] J. Schwarz-Linek, C. Valeriani, A. Cacciuto, M. E. Cates, D. Marenduzzo, A. N. Morozov, and W. C. K. Poon, Proc. Natl. Acad. Sci. USA 109, 4052 (2012).

[15] T. Qiu, T.-C. Lee, A. G. Mark, K. I. Morozov, R. Münster, O. Mierka, S. Turek, A. M. Leshansky, and P. Fischer, Nat. Commun. 5, 5119 (2014).

[16] L. Zhu, E. Lauga, and L. Brandt, J. Fluid Mech. 726, 285 (2013).

[17] E. E. Riley and E. Lauga, Europhys. Lett. 108, 34003 (2014).
[18] G. J. Elfring and E. Lauga, in Complex Fluids in Biological Systems, edited by S. E. Spagnolie (Springer Science+Business Media, New York, 2015), pp. 283-317.

[19] J. R. Gomez-Solano, A. Blokhuis, and C. Bechinger, Phys. Rev. Lett. 116, 138301 (2016).

[20] J. Berner, B. Müller, J. R. Gomez-Solano, M. Krüger, and C. Bechinger, Nat. Commun. 9, 999 (2018).

[21] J. Shi and T. R. Powers, Phys. Rev. Fluids 2, 123102 (2017).

[22] J. S. Lintuvuori, A. Würger, and K. Stratford, Phys. Rev. Lett. 119, 068001 (2017).

[23] J. Toner, H. Löwen, and H. H. Wensink, Phys. Rev. E 93, 062610 (2016).

[24] S. Zhou, A. Sokolov, O. D. Lavrentovich, and I. S. Aranson, Proc. Natl. Acad. Sci. USA 111, 1265 (2014).

[25] O. D. Lavrentovich, Curr. Opin. Colloid Interface Sci. 21, 97 (2016).

[26] P. C. Mushenheim, R. R. Trivedi, H. H. Tuson, D. B. Weibel, and N. L. Abbott, Soft Matter 10, 88 (2014).

[27] S. Hernàndez-Navarro, P. Tierno, J. Ignés-Mullol, and F. Sagués, IEEE Trans. Nanobiosci. 14, 267 (2015).

[28] M. S. Krieger, M. A. Dias, and T. R. Powers, Eur. Phys. J. E 38, 94 (2015).

[29] M. S. Krieger, S. E. Spagnolie, and T. R. Powers, Soft Matter 11, 9115 (2015).

[30] R. R. Trivedi, R. Maeda, N. L. Abbott, S. E. Spagnolie, and D. B. Weibel, Soft Matter 11, 8404 (2015).

[31] O. Chepizhko and F. Peruani, Phys. Rev. Lett. 111, 160604 (2013).

[32] G. Volpe, I. Buttinoni, D. Vogt, H.-J. Kümmerer, and C. Bechinger, Soft Matter 7, 8810 (2011).

[33] A. Morin, N. Desreumaux, J.-B. Caussin, and D. Bartolo, Nat. Phys. 13, 63 (2017).

[34] M. S. Krieger, S. E. Spagnolie, and T. R. Powers, Phys. Rev. E 90, 052503 (2014). 
[35] B. van der Meer, L. Filion, and M. Dijkstra, Soft Matter 12, 5630 (2016).

[36] A. T. Brown, I. D. Vladescu, A. Dawson, T. Vissers, J. SchwarzLinek, J. S. Lintuvuori, and W. C. K. Poon, Soft Matter 12, 131 (2016).

[37] The only exception to this would be smectics in aerogels. In these systems, the smectic can lose momentum to the aerogel mesh. However, in this case the physics is quite different, due to the effects of the disorder of the aerogel on the smectic. See T. Bellini, L. Radzihovsky, J. Toner, and N. K. Clark, Science 294, 1074 (2001), for a discussion of these systems.

[38] P. G. de Gennes and J. Prost, The Physics of Liquid Crystals (Clarendon Press, Oxford, 1995).

[39] The full smectic elastic Hamiltonian contains anharmonic terms which are known to significantly change the scaling of the smectic correlations at long distances. However, the length scale at which these effects become important is also astronomically large in most real systems. Hence we will ignore these effects here. The theory of these effects was worked out by G. Grinstein and R. A. Pelcovits, Phys. Rev. Lett. 47, 856 (1981).

[40] P. C. Martin, O. Parodi, and P. S. Pershan, Phys. Rev. A 6, 2401 (1972).

[41] W. Helfrich, Z. Naturforsch. 33a, 305 (1978).

[42] J. S. Lintuvuori and M. R. Wilson, J. Chem. Phys. 128, 044906 (2008).

[43] M. M. Tirado, J. G. de la Torre, and C. L. Martinez, J. Chem. Phys. 81, 2047 (1984).

[44] K. Drescher, J. Dunkel, L. H. Cisneros, S. Ganguly, and R. E. Goldstein, Proc. Natl. Acad. Sci. USA 108, 10940 (2011).

[45] S. Hernàndez-Navarro, P. Tierno, J. Ignés-Mullol, and F. Sagués, Soft Matter 9, 7999 (2013). 